Manfred Nagl (Ed.)

\title{
Graph-Theoretic Concepts in Computer Science
}

21st International Workshop, WG '95

Aachen, Germany, June 20-22, 1995

Proceedings 


\section{Contents}

VC-Dimensions for Graphs

E. Kranakis, D. Krizanc, B. Ruf, J. Urrutia, G. J. Woeginger

Finding and Counting Small Induced Subgraphs Efficiently

T. Kloks, D. Kratsch, H. Müller

On the Isomorphism of Graphs with Few $P_{4} s$

L. Babel, S. Olariu

A Dynamic Algorithm for Line Graph Recognition

D.G. Degiorgi, K. Simon

Incremental Hive Graph

F. d'Amore, R. Giaccio

Planarization of Graphs Embedded on Surfaces

H. N. Djidjev, S. M. Venkatesan

Complexity and Approximability of Certain

Bicriteria Location Problems

S. O. Krumke, H. Noltemeier, S. S. Ravi, M. V. Marathe

On Termination of Graph Rewriting

D. Plump

A Uniform Approach to Graph Rewriting: The Pullback Approach M. Bauderon

Visualizing Two- and Three-Dimensional Models of Meristematic Growth

F. D. Fracchia

Graph-Theoretical Methods to Construct

Entity-Relationship Databases

S. Hartmann 
An Approximation Algorithm for 3-Colourability

I. Schiermeyer

The Malleability of $T S P_{2 O p t}$

S. Fischer, L. Torenvliet

Non-Oblivious Local Search for Graph and Hypergraph

Coloring Problems

P. Alimonti

On Interval Routing Schemes and Treewidth

H. L. Bodlaender, R. B. Tan, D. M. Thilikos, J. van Leeuwen

Highly Fault-Tolerant Routings and Diameter Vulnerability

for Generalized Hypercube Graphs

K. Wada, T. Ikeo, K. Kawaguchi, W. Chen

Hot-Potato Routing on Multi-Dimensional Tori

F. Meyer auf der Heide, M. Westermann

On Devising Boolean Routing Schemes

M. Flammini, G. Gambosi, S. Salomone

Toward a General Theory of Unicast-Based

Multicast Communication

B. D. Birchler, A.-H. Esfahanian, E. Torng

Optimal Cutwidths and Bisection Widths of 2- and 3-Dimensional Meshes

J. Rolim, O. Sykora, I. Vrt'o

Searching for Faulty Leaves in Binary Trees

P. Damaschke

NC Algorithms for Partitioning Planar Graphs into Induced Forests and Approximating NP-Hard Problems

Z.-Z. Chen, X. He

Efficient Parallel Modular Decomposition

E. Dahlhaus 
Modular Decomposition of Hypergraphs

P. Bonizzoni, G. Della Vedova 303

Partition Coefficients of Acyclic Graphs

J. L. Pfaltz

Sub-Cubic Cost Algorithms for the All Pairs Shortest Path Problem

T. Takaoka

Diametral Path Graphs

J.S. Deogun, D. Kratsch

Chordal Graphs and their Clique Graphs

P. Galinier, M. Habib, C. Paul

A Compact Data Structure and Parallel Algorithms for Permutation Graphs

J. Gustedt, M. Morvan, L. Viennot

372

Homogeneously Orderable Graphs and the Steiner Tree Problem A. Brandstädt, F. F. Dragan, F. Nicolai 381

List of Participants 397

Authors' Index 403

List of WG Proceedings 405 